\title{
How to Organize an OR Workshop: the AIROYoung Experience
}

\author{
Rossana Cavagnini ${ }^{1}$. Veronica Dal Sasso ${ }^{2}$. Valentina Morandi ${ }^{3}$ (D) . \\ Alice Raffaele ${ }^{4}$
}

Received: 15 June 2020 / Accepted: 28 September 2020 / Published online: 13 November 2020

(C) The Author(s) 2020

\begin{abstract}
AIROYoung is the Young Researchers Chapter of AIRO (Italian Association of Operations Research). In this work, we focus on the AIROYoung Workshop, an annual event which has been reuniting young researchers constituting this community from 2017. Describing all the editions of the initiative, we highlight the main guidelines we have been following so far to organize it (e.g., no registration fee, grants for free accommodation, no parallel sessions, and a look to sustainability). We analyze surveys filled by participants to present qualitative and quantitative data about our workshops and to show how interest and attendance have increased through the years. We talk about some innovations we have introduced to improve quality (e.g., the $\mathrm{PhD}$ school "from young researchers to young researchers" and the pitch talk session). We then share our personal opinion and, last but not least, we invite young researchers to join in the preparation of the next AIROYoung Workshops.
\end{abstract}

Keywords OR community · AIROYoung · Workshop · Organization skills

\section{Introduction}

AIROYoung (AY) is the Young Researchers Chapter of AIRO (Italian Association of Operations Research). AY is not just a web platform [1], but a real concrete community composed of many young researchers which, once a year, meet in a beautiful Italian city.

Electronic Supplementary Material The online version of this article (https://doi.org/10.1007/s43069-020-00031-2) contains supplementary material, which is available to authorized users.

This article is part of the Topical Collection on The AIROYoung Experience: Operations Research for Young Enthusiasts

Valentina Morandi

valentina.morandi@unibz.it

Extended author information available on the last page of the article. 
After its 4th edition, the AIROYoung Workshop (AYW) has come to be considered an eagerly anticipated annual event, as testified by attendees coming back year after year. But how did it originate? There are a few examples around Europe of events organized by young researchers for young researchers. Maybe the most known is the Student Conference on Operations Research (SCOR) [2], held in Nottingham every 2 years. Recently, the Spanish Society of Statistics and Operations Research (SEIO) has introduced a similar format [3]. AY was launched during the 46th Annual Conference of AIRO in 2016. At that time, the founders had not an initiative like this in mind yet. They only had a rough idea of what AY was meant to be, that is, a way to connect PhD students all over Italy and also from abroad. But within the end of the conference, the enthusiasm spread also to other young researchers. In fact, it is common in Italy to find $\mathrm{PhD}$ students in Operations Research (OR) affiliated to different university departments, such as Mathematics, Statistics, Economics or Management. Hence, they are part of small research groups with, sometimes, no fellow PhD students. The opportunity to meet young colleagues was tantalizing and lead to the question: "And now? What are we going to do? When are we meeting again?". At that time, this sounded almost like a joke: "Let's organize a workshop!". However, the idea was good, and after some thoughts it seemed to be doable.

And here we are. If you are curious about these workshops, then read along. On behalf of AY, in this paper, we give an overview of this recurring initiative. In Section 2, we briefly describe the previous editions with a special focus on this year's workshop. Section 3 highlights the guidelines we have been following so far. Section 4 provides analytical insights on the numbers that characterized the AYWs. In Section 5, we describe the innovative aspects and features of the two latest editions. We share what we particularly liked about organizing this workshop in Section 6 and we draw our conclusions in Section 7.

\section{Our Workshops}

The annual AYW was born as a workshop for young OR enthusiasts, with a particular attention from the very beginning to reduce costs and to provide a free of charge workshop, to let $\mathrm{PhD}$ students and young scientists enjoy the AY community without using their funds.

The main goals of the AYWs are fostering collaboration among young researchers in OR field and providing them the opportunity to share their work in a relaxed and young environment.

The 1st AYW [4] was held in Rome in 2017 and it had a remarkable success with high-level invited talks and a good level of participation. Thirty-five participants with different nationalities enrolled in the workshop and enjoyed two high-quality plenary, held by Justo Puerto (University of Sevilla, Spain) and Maria Grazia Scutellà (University of Pisa, Italy). At that time, we were already able to provide travel allowance for 9 attendees.

In 2018, the 2nd AYW [5] was held in the beautiful city of Rende (Cosenza) and, even though Europe was under a huge snow storm, a significant number of 
attendees was able to join the workshop and most of them were covered with free accommodation. The event was a success and two great scientists in OR field, Geir Hasle (SINTEF, Norway) and Roberto Roberti (Vrije Universiteit Amsterdam, The Netherland), held plenary lectures. The workshop also hosted an invited lecture from the industry by Pierluigi Montanari (NTT Data, Italy), about real-world supply chain.

We moved back to Rome in 2019 for the 3rd AYW [6], with a stunning new adding: the 1st PhD School, i.e., the first school for OR PhD students with tutorials and lectures given by AY researchers. As for the workshop, the school was free of charge and we provided free accommodation to a subset of the attendees. Several plenary talks were offered alongside practical and theoretical tutorials held by young experienced researchers. Plenary talks were held by well-known names in the OR field: Teodor G. Crainic (Université du Quebec à Montréal, Canada), Endre Boros (Rutgers University, NJ, USA), Paolo dell'Olmo (Sapienza University, Rome), and Laura Palagi (Sapienza University, Rome). From this edition, also sponsors have been starting to present, in about $10 \mathrm{~min}$ each, how they put OR into practice, thus promoting collaborations between the academic world and the industry. This time 59 participants attended the workshop and the school, coming from 8 different countries around the world. They were enthusiastic about both the format and the contents of the school, as assessed by the anonymous survey we used to enhance the services to provide in subsequent workshops.

In 2020, we moved to the deep North of Italy for the 4th AYW [7], in the border city of Bozen (Fig. 1). As a novelty for the workshop, we decided to leave room also for academics and practitioners coming from data science and machine learning, in order to let AIROYoungers be aware of the new findings in these multidisciplinary

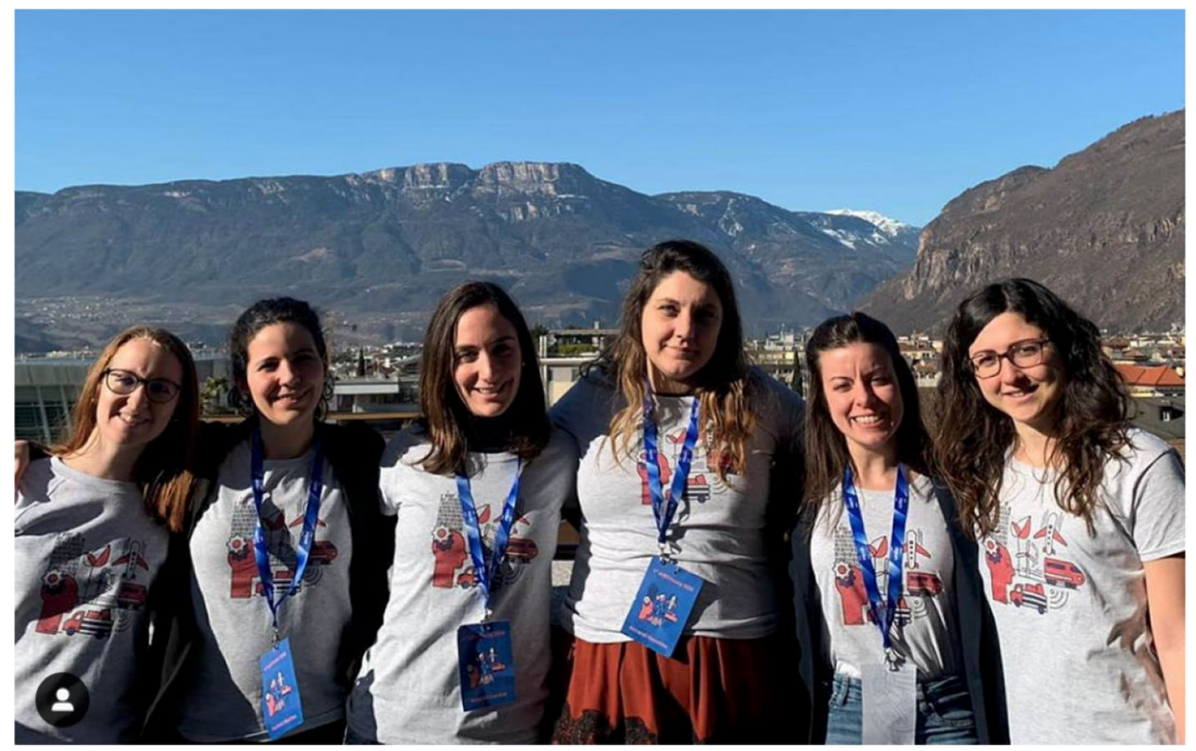

Fig. 1 The 4th AYW organizing committee in Bozen 
fields. We also organized a pitch talk round table session for early stage PhD students. We organized very high-quality plenary talks, held by David Pisinger (DTU, Copenhagen) and Claudia Archetti (ESSEC Business School, Paris). Moreover, Tullia Padellini (Imperial College, London) gave an exceptional plenary talk on the crossing point between OR and Data Science. Once again, participants were more than 50, coming from several countries around Europe helding more than 20 session talks. As in the previous years, the 4th AYW was able to attract funds from academic and industrial institutions in recognition of the service done to the OR community and we used them to provide, as usual, free accommodation and high-quality services, while still being free of charge. And, if that is not enough, the AYW continues to attract researcher of national and international OR groups and young practitioners too.

Here follow the feedback of the two plenary speakers of this year's edition which confirm the opinions given by participants.

David Pisinger "The AY conference in Bozen was of a high academic standard with many interesting talks and an excellent social program. One could feel a willingness for cooperation and knowledge exchange that you seldom meet at other conferences. A must-go for PhD students."

Claudia Archetti "I had the privilege and the pleasure to be invited as plenary speaker at the $4^{\text {th }}$ AYW in Bozen. It was indeed a wonderful experience: a great group of talented, passioned and super-active young OR researchers, eager to learn and exchange new challenging ideas. I was especially impressed by the enthusiasm and the energy of the members of the AY Board: five fantastic young OR women who will certainly leave their mark in our scientific community. A special thanks to all of them and in particular to the local organizer, Valentina Morandi, for the great experience, the fantastic atmosphere and the excellent scientific quality of the workshop. Unforgettable!"

As a side, we plan engaging social activities during all the events. For example, this last edition we organized both a social dinner and a guided tour of the city of Bozen. Furthermore, and this is beyond academic purposes, during the AYWs, several collaborations were born.

Now the AY community is ready for the next workshop, the 5th one, which will be again organized with the goals of constantly improving the quality of the workshop itself and of strengthening relations among OR researchers and related fields.

\section{Guidelines}

In this section, we want to describe the focal points we considered while organizing the workshops so far. While we are strongly convinced that these guidelines are the ones we want to pursue, the way in which we achieve them is not definitive, as we are always trying to improve and to be as inclusive as possible. 
AIROYoung established the AYW to give the opportunity to $\mathrm{PhD}$ students and early researchers to participate to a national event and cultivate their academic network. Being aware of the fact that funds for conferences are limited, and that often they are carefully administrated, we make a point in ensuring that no participation fee is required. This has been possible, in all the editions so far, thanks to the generosity of our sponsors and the help we got from full professors. Along the same line, we always make sure to be able to offer travel compensation, in the form of free accommodation, for a subset of the participants belonging to the AY network. As far as now, we allocated the free accommodation on a first-come-first-served basis. This modus operandi has endorsed the actual lack of funds of PhD students: the grants are usually assigned in no more than a couple of hours from the registration opening.
Guidelines in pills
1. No registration fee
2. Limited grants for free
2. accommodation
3. Cutting edge plenary
2. talks
4. No parallel sessions
5. Catching social events
6. Plastic free!

To mitigate the endemic risk of free events that is no-showing people, we ask for a guarantee for each participant's commitment in the form of a proof of travel arrangements. It proved to be a valid system so far, as usually, in case of a real interest in the event, travel arrangements are taken in advance in any case.

We always try to provide a worthy academic experience by inviting prominent professors (to which we are extremely grateful) to give plenary session seminars.

An important goal of the workshops is to provide opportunities for networking, either by getting to know participants' research topics or by having a chat and a pint of beer, or two, together. For this reason, we decided not to organize parallel sessions, but to give the participants the opportunity to listen to all the talks. We consider this a key feature of our workshops as PhD students and young researchers may not be yet aware of fellow researchers studying the same topic. Moreover, it is also a good opportunity to broaden our set of interests, with methodologies and applications that are not so widely known. A fundamental contribution to networking is provided by social events, which usually include a social dinner and a visit to an historical site of interest. Sometimes we have been able to partially cover the expenses for social events, but, in principle, they are the only things we do not guarantee to be cost-free for participants.

Last but not least, as part of the young generation, we feel we cannot neglect the increasing awareness on environmental crisis. Hence, from the $3 \mathrm{rd}$ AYW, we are plastic-free! This means that not only we have removed plastic cups from the coffee breaks but we also pay more attention on the selection of gadgets. 


\section{Numbers and Charts}

AIROYoung already counts four editions of its workshops and data are now available to draw insights. In this section, we first present the factual data of AYWs, and then, we focus on the analysis of the surveys given to the participants.

\subsection{AYW Data Analysis}

The data collected so far can be used to draw insights on the participation features and on the financial aspects of the previous AYWs.

Figure 2 summarizes the most interesting data concerning the participation. The number of participants has increased over time, as well as the percentage of young women researchers involved. Even if AIROYoung is an Italian association, participants are far from being all Italians. More and more young researchers of institutions from all over Europe and abroad (Canada and China) are joining the workshops and participating to AIROYoung activities. Specifically, during the 1st AYW, participants came from only two countries, while in the latest three editions, the participants' countries were five, seven, and nine, respectively. In addition to networking, AYWs are very active from the scientific point of view. Figure $2 b$ seems to contradict this statement because the number of presentations during standard sessions has decreased over the years. However, this is a consequence of the introduction of new special scientific initiatives that reduced the time available for standard sessions.

Data concerning AYWs' financial aspects are displayed in Fig. 3. Sponsors play a fundamental role for the success of these events. During the years, we have always attracted funds from industrial and academic sponsors, which is a signal of interest in OR community. Starting from the 1 st AYW, which was funded by the EURO [8], AIRO [9], and Sapienza University [10], numerous other sponsors have joined the list. Specifically, the 2nd AYW was sponsored by the NTT Data [11], Link [12], Optit [13], AIRO, VeRoLog [14], WeSmart [15], and the University of Calabria [16]; the 3rd AYW by Optit, AIRO, ACTOR [17], Optrail [18], Vattenfall [19], GERAD [20], CIRRELT [21], CORS [22], the Montreal OR Student Chapter (MORSC) [23], and Sapienza University. For the 4th AYW, Optit, ACTOR, AIRO, and the Free University of Bozen [24] have sponsored the event. Relevant funds have also come from some AIROYoungers who decided to devolve prizes received for their research activities entirely to AY. In addition, food-related companies have sponsored our workshops: Nicola Colavolpe \& C. S.N.C [25] and Caffé Iaquinta [26] (2nd AYW), Loacker S.p.A [27] (4th AYW). Finally, thanks to the constant support of our sponsors, we have been able to provide an increasing number of grants to participants all over the years and we hope this trend will be replicated in the future.

\subsection{AYW Survey Analysis}

From the 3rd AYW, surveys have been sent to all participants, who are welcome to express their opinions and suggestions in anonymous form. Collecting feedback 


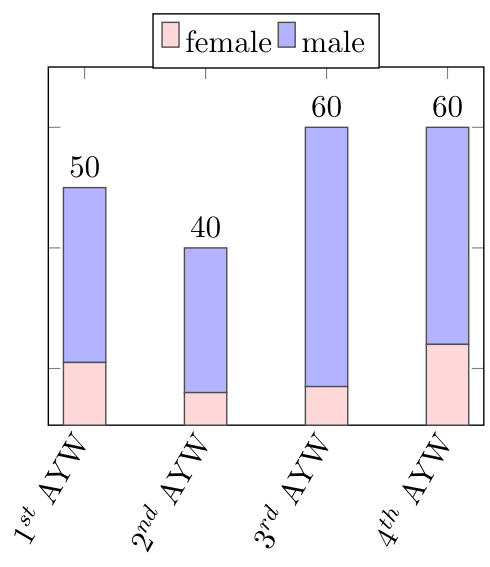

(a) Number of participants by gender and by AYW

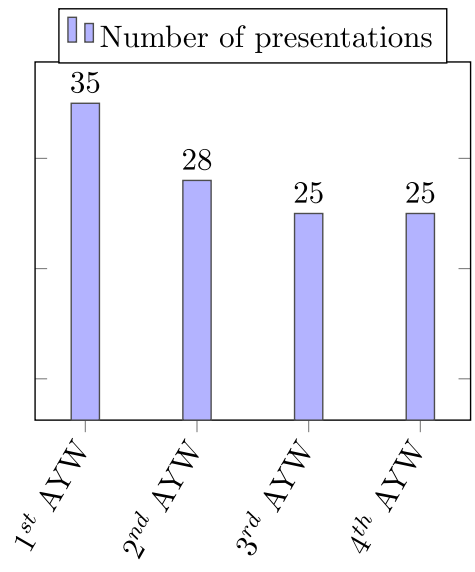

(b) Number of presentations (invited speakers excluded) by AYW

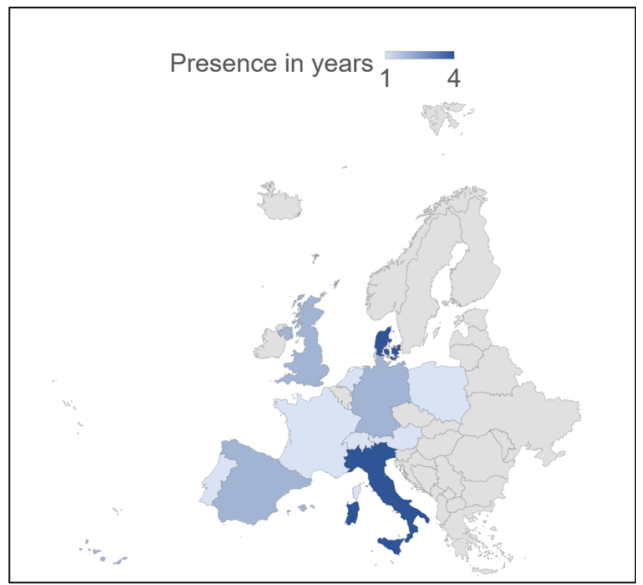

(c) Presence (in number of years) by European country (outside Europe: Canada (1), China (1))

Fig. 2 a-c Participation trend analysis

has helped us to improve the organization of these events during the years and to keep participants' satisfaction levels high. These surveys cover different aspects. In particular, AIROYoung is working hard to improve and assess the impact of its workshops through the following topics: (1) the effectiveness of the communication strategy to advertise the workshop, (2) the quality of the organizational aspects of the workshop, (3) its scientific soundness.

The results in Fig. 4b show that personal communication and the AIROYoung mailing list are the most effective advertising channels for the workshops. Moreover, 


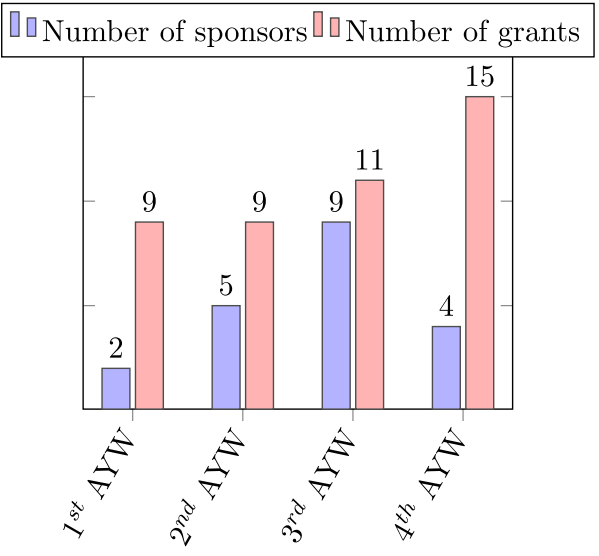

(a) Number of sponsors and number of grants by AYW

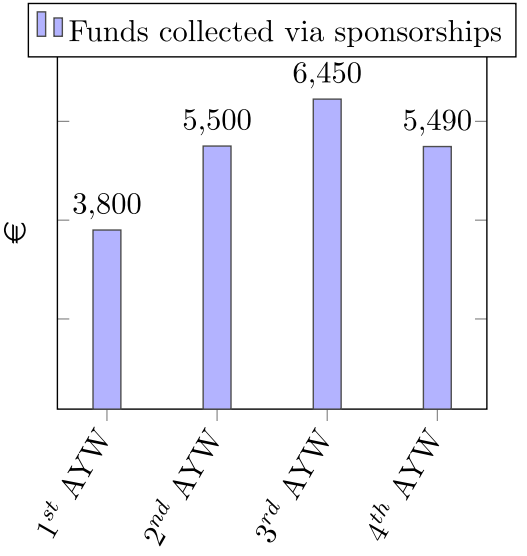

(b) Funds collected via sponsorships by AYW

Fig. 3 a, b Financial trend analysis by AYW

the AIROYoung mailing list has been more effective for the 4th workshop compared with the 3rd one.

The quality of the general organization (Fig. 4a) has registered high scores for both workshops. Specifically, for the 4 th workshop, there has been an improvement in the classroom and accommodation organization (for participants with a grant), while the 3rd one has a higher score for the organization of social events and coffee breaks. Concerning the scientific soundness (Fig. 4c), during the last workshop, we registered improvements both in the quality of the standard seminar sessions and in the attractiveness of the presented topics.

Finally, in the last AYW, we introduced two new features, i.e., a special session on "Optimization and Data Science" and a pitch talk session. Figure 5a presents the satisfaction levels for the "Optimization and Data Science" session. The $96.16 \%$ of the interviewees show interest in this new session, with only a small number of the participants not satisfied at all. Concerning the pitch talk session, Fig. 5b shows that participants are in general enthusiast and they suggest to move this session at the beginning of the workshop to have the chance to know each other's research topics during the first day. Considering the positive feedback, these are two initiatives which deserve more attention in the future, and we will dedicate more efforts in improving them.

\section{Innovative Parts}

Even if the editions of the AYW so far have been based on the guidelines described in Section 3, we are always looking at how we can integrate and innovate their structure, trying to make the event more interesting and appealing to our participants. We asked ourselves: "What do young researchers need, at the beginning of their careers?". Actually there is not just one exact answer, but we considered several aspects. 


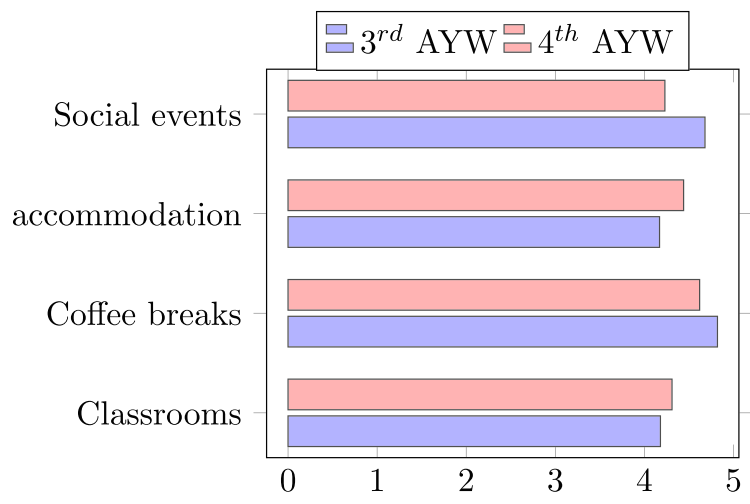

(a) Average results for the general organization quality for the $3^{\text {rd }}$ AYW and $4^{\text {th }}$ AYW (0: not sufficient - 5: perfect)

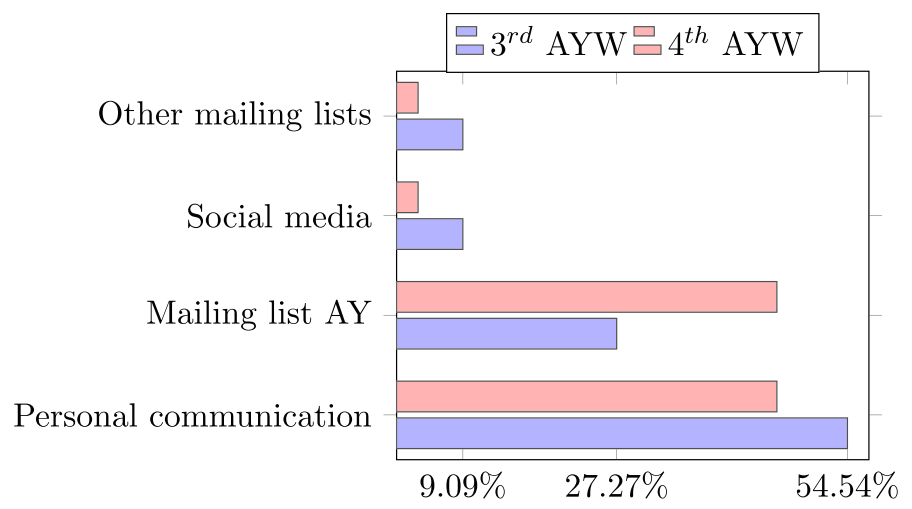

(b) Results to the question "How did you know about the AYW?" for the $3^{\text {rd }} \mathrm{AYW}$ and $4^{\text {th }} \mathrm{AYW}$

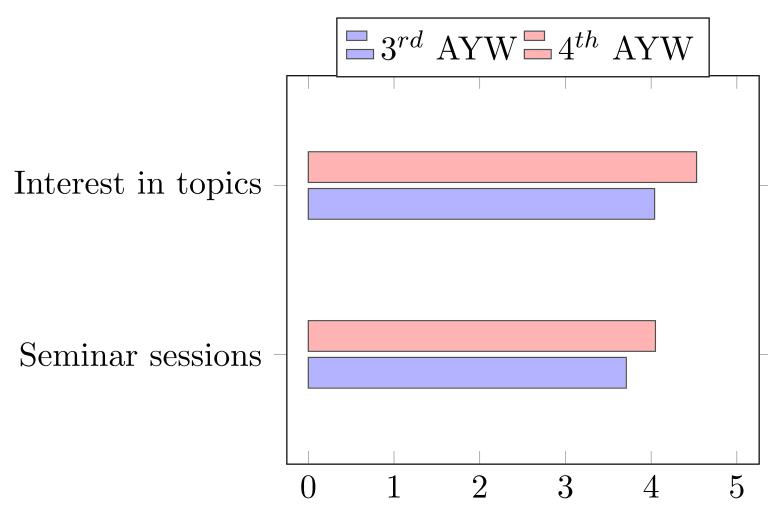

(c) Average results for the seminar sessions organization and the interest in the topics (0: not sufficient -5 : perfect)

Fig. 4 a-c General opinions on AYWs 


\section{Interesting}

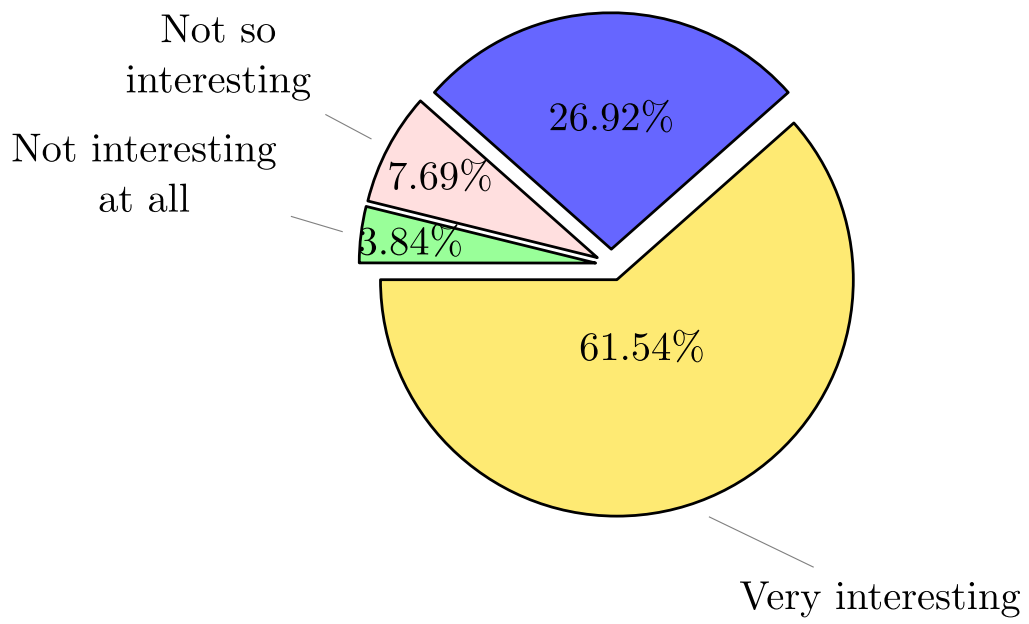

(a) Results for the "Optimization and Data Science" sessions

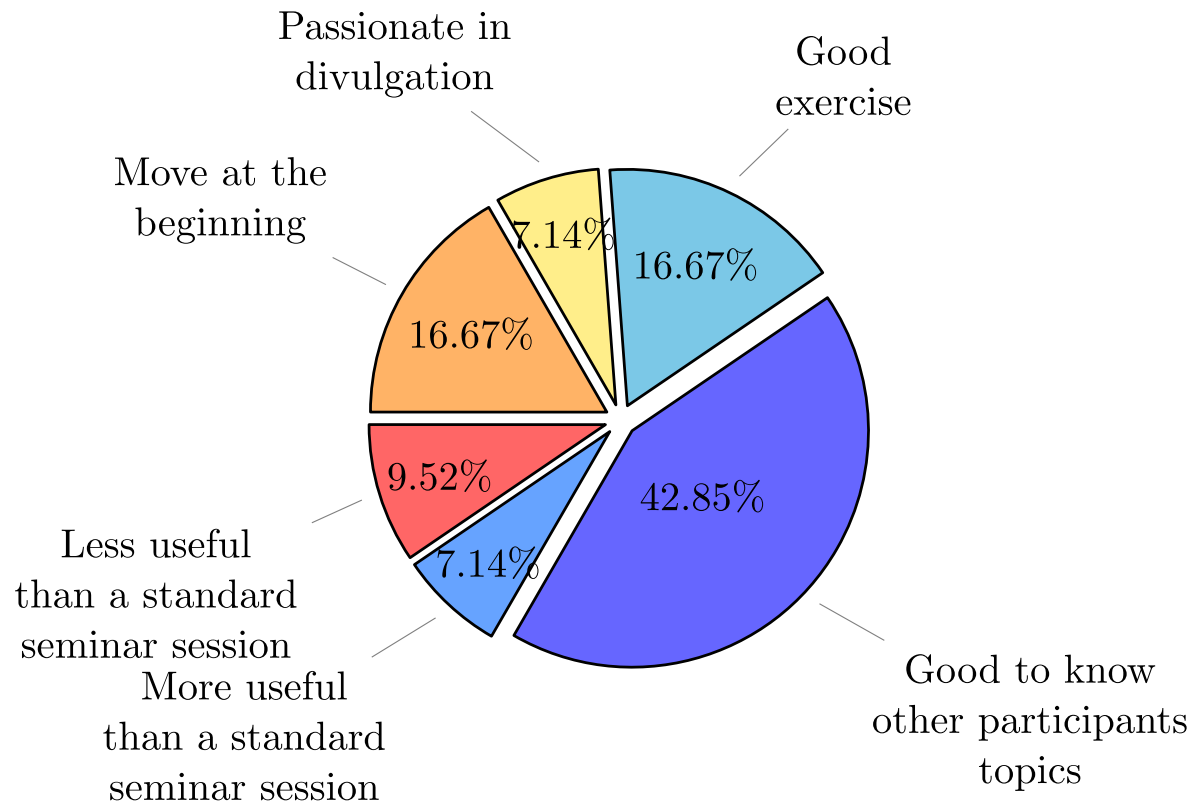

(b) Results for the pitch talk session evaluation

Fig. 5 a, b Opinions on 4th AYW special sessions 
$\mathrm{PhD}$ students, in particular the first year ones, may not have a wide knowledge of the research topics and methodologies either because of their specific background (if they stick to the same topic studied in previous research experiences) or because they dive into something completely new. In both cases, and since OR is at the intersection of mathematics, economics, computer science, management science, and several other disciplines, one will probably have to learn new tools and techniques, enriching her/his set of skills and abilities. Thus, our first answer was the proposal of the 1st AY School, during the 3rd AYW. Beyond the two plenary lectures, we offered four tutorials on the following topics: two solvers for multicriteria linear optimization; an introduction to the modern programming language Julia; how to expose C++ classes to Python; last but not least, how to transform a mathematical representation into code. The distinctive feature was that all tutorials were taught by other young researchers. This swept away some shyness and reserve, because the perceived distance between teachers and students was almost zero.

This was also one of the main reasons we invited Tullia Padellini, a young Research Associate in Spatial Statistics and Epidemiology at the Imperial College in London. At the 4th AYW, she talked about possible bridges between OR and Data Science. Not only by famous experts in the field: young researchers can also be inspired by peers steps ahead in the research path. Moreover, we had the chance to discuss about the interplay between different areas of mathematics, showing participants that they can mix and put together disparate resources to solve their problems.

One of the main effects of our pitch talk session, still during the 4th AYW, was the disappearance of embarrassment, awkwardness, and fears. After writing down what OR means to them and for what purposes they use it, all participants had exactly 1 min each to present their projects. Being able to summarize and highlight the very essential features of a project is a very precious competence for a researcher. This exercise allowed them to practice and receive suggestions and feedback from each other, while connecting and starting to share their research, which was the main purpose of the session and another answer to the question above. To this purpose, the special issue is a novelty for us and a chance not to miss for them.

Finally, another thing researchers should do is divulging their work to the public. That is why we recorded a few short videos during the pitch talk, publishing them on our social network accounts, trying to spread what OR can do.

\section{Our Personal Opinion}

After all discussed in the previous sections, one may think that organizing a workshop could be challenging, figuring out what needs to be scheduled and making sure everything is ready on time. And for sure it is, but also definitely worthy. Indeed, as organizers of the 4th AYW, we all agree that this has been an unforgettable and rewarding experience, in several ways.

We had the opportunity to learn how to interact with all actors involved in such kind of events (i.e., the university, the organizing committee, sponsors, and firms for social activities). We were able to widen our networks and get to know other young 
researchers and professionals, giving a boost to our CVs and improving the visibility of the involved university too.

Furthermore, from a formative point of view, we developed collaborative, problem solving, and management skills, respecting deadlines and working in a very supportive team. Sharing thoughts and opinions, being part of a group, and contributing to its development: this has played a crucial role in the success of the workshop. In the end, we have found ourselves proudly saying "We made it!", all together as a team but also individually, each one of us accomplishing her own tasks.

Anyway, this does not imply we are completely satisfied with our current strategy: we are constantly striving to improve our AYWs and always open to new ideas and suggestions, hopefully coming from other enthusiast AIROYoungers who would like to contribute.

\section{Conclusion}

The AIROYoung Workshops are of great importance for the entire OR community for several reasons. First, they involve young OR enthusiasts and researchers in a relaxed environment, where the networking and the discussion about research ideas are made easier. Since the attendees come from several countries around the world, the AYWs are far from being initiatives with only a national flavor, but rather events with an international relevance. As the discussion among researchers is strongly encouraged, the knowledge of other participants' research topics is enhanced and several collaborations were born.

Organizing the AYW is itself a very formative experience. Being researchers also means having the ability of organizing events, collaborating with other colleagues, and searching for new ideas and topics that represent trends in our field. If you are willing to organize an AYW, you would be involved in all these activities that may also help you to get known in the OR community. Moreover, you will contribute to foster collaboration and research among young OR researchers. Some of them will likely be your colleagues, co-authors, or collaborators in the future. Thus, we believe that the AYW organization experience can be easily seen as your futureproof time investment. Through the years, the organization has been well-proven and the past committees have always been helping the new ones in several ways: planning, accounting matters, searching for funds, advertising strategies, social networks, and many other subjects. Consequently, do not hesitate to contact the AY board for developing your ideas in the next workshop. This will be an amazing experience for you.

Funding Open access funding provided by Libera Universit di Bolzano within the CRUI-CARE Agreement.

\section{Compliance with Ethical Standards}

Conflict of Interest The authors declare that they have no conflict of interest. 
Open Access This article is licensed under a Creative Commons Attribution 4.0 International License, which permits use, sharing, adaptation, distribution and reproduction in any medium or format, as long as you give appropriate credit to the original author(s) and the source, provide a link to the Creative Commons licence, and indicate if changes were made. The images or other third party material in this article are included in the article's Creative Commons licence, unless indicated otherwise in a credit line to the material. If material is not included in the article's Creative Commons licence and your intended use is not permitted by statutory regulation or exceeds the permitted use, you will need to obtain permission directly from the copyright holder. To view a copy of this licence, visit http://creativecommonshorg/licenses/by/4. $0 \%$.

\section{References}

1. AIROYoung Researchers Chapter. Link: https://www.airoyoung.org. Accessed: 2020-06-01

2. Student Conference on Operations Research. Link: http://scor18.com/. Accessed: 2020-05-29

3. Spanish Young Statisticians and Operational Researchers Meeting. Link: https://eventos.ucm.es/ 17713/detail/2nd-spanish-young-statisticians-and-operational-researchers-meeting.html. Accessed: 2020-05-29

4. AIROYoung Researchers Chapter. 1st AIROYoung Workshop. Link: https://workshop.airoyoung.org/ 2017. Accessed: 2020-06-01

5. AIROYoung Researchers Chapter. 2nd AIROYoung Workshop. Link: https://workshop.airoyoung.org/ 2018. Accessed: 2020-06-01

6. AIROYoung Researchers Chapter. 3rd AIROYoung Workshop. Link: https://workshop.airoyoung.org/ 2019. Accessed: 2020-06-01

7. AIROYoung Researchers Chapter. 4th AIROYoung Workshop. Link: https://www.unibz.it/en/ events/132924-4th-airoyoung-workshop-optimization-at-the-crossroads-of-italy-and-north-europe. Accessed: 2020-06-01

8. Association of European Operational Research Societies. Link: https://www.euro-online.org. Accessed: 2020-06-10

9. Associazione Italiana di Ricerca Operativa (AIRO). Link: https://www.airo.org. Accessed: 2020-0606

10. Sapienza UniversitÃ? di Roma. Link: https://www.uniroma1.it/it/pagina-strutturale/home. Accessed: 2020-06-06

11. NTT DATA. Link: https://it.nttdata.com. Accessed: 2020-06-06

12. Link Telecomunicazioni. Link: www.link.cs.it. Accessed: 2020-06-09

13. Optit s.r.1. Link: https://www.optit.net. Accessed: 2020-06-06

14. Working Group on Vehicle Routing and Logistics Optimization within EURO (VeRoLog). Link: http://www.verolog.eu. Accessed: 2020-06-06

15. WeSmart. Link: http://www.wesmart.it/. Accessed: 2020-06-06

16. Universit $\tilde{A}$ ? della Calabria. Link: https://www.unical.it/portale/. Accessed: 2020-06-06

17. ACTOR s.r.1. Link: http://www.actorventure.com. Accessed: 2020-06-06

18. Optrail s.r.1. Link: http://optrail.com. Accessed: 2020-06-06

19. Vattenfall AB. Link: https://group.vattenfall.com. Accessed: 2020-06-06

20. Group for Research in Decision Analysis (GERAD). Link: https://www.gerad.ca/en. Accessed: 202006-06

21. Centre interuniversitaire de recherche sur les reseaux d'entreprise, la logistique et le transport (CIRRELT). Link: https://www.cirrelt.ca. Accessed: 2020-06-06

22. Canadian Operational Research Society (CORS). Link: https://www.cors.ca. Accessed: 2020-06-06

23. Montreal Operations Research Student Chapter (MORSC). Link: https://www.mtl-students.com. Accessed: 2020-06-06

24. Free University of Bozen-Bolzano. Link: https://www.unibz.it/. Accessed: 2020-06-06

25. Nicola Colavolpe \& C. S.N.C. Link: https://colavolpe.com/. Accessed: 2020-06-09

26. Caffé Iaquinta S.r.1. Link: https://caffeiaquinta.shop/. Accessed: 2020-06-09

27. Loacker S.p.A. Link: https://www.loacker.com/int/it/. Accessed: 2020-06-09

Publisher's Note Springer Nature remains neutral with regard to jurisdictional claims in published maps and institutional affiliations. 


\section{Affiliations}

\section{Rossana Cavagnini ${ }^{1}$ - Veronica Dal Sasso ${ }^{2}$. Valentina Morandi ${ }^{3}$ (D) . Alice Raffaele 4}

Rossana Cavagnini

cavagnini@dpo.rwth-aachen.de

Veronica Dal Sasso

veronica.dalsasso@optrail.com

Alice Raffaele

alice.raffaele@unitn.it

1 Deutsche Post Chair - Optimization of Distribution Networks, RWTH Aachen University, Aachen, Germany

2 Optrail s.r.1., Rome, Italy

3 Faculty of Science and Technology, Free University of Bozen-Bolzano, Bolzano, Italy

4 Department of Mathematics, University of Trento, Trento, Italy 